\title{
Population estimates from patient registers held by British family health services authorities
}

\author{
R M Haynes, A A Lovett, G Bentham, J S Brainard, S H Gale
}

Epidemiologists and purchasers or providers of health care in the UK need access to detailed and up to date information on the distribution of population, but the usefulness of the decennial census decreases with time, while annual midyear population estimates do not provide age breakdowns below the scale of local and health authority districts. Patient registers currently maintained by family health service authorities (FHSAs) are an alternative source of population estimates but their accuracy has been questioned because of the problems of delay in removing outdated records and non-registration, particularly in inner city areas. ${ }^{1}$ There is also concern about undercounting in the 1991 census. $^{2}$ Here we compare population estimates in broad age-sex categories from two patient registers with estimates derived from the 1991 census. Fuller details and a comparison at census ward level are published elsewhere. ${ }^{3}$

Methods and results: Partial and anonymous patient records current on 21 April 1991 (census day) were obtained from Norfolk and Suffolk FHSAs and patients were grouped into broad age-sex categories. 1991 census counts of the usually resident population by age and sex were obtained for the same areas. ${ }^{4}$ Long stay hospital patients, prison residents, armed forces personnel, and students studying elsewhere were subtracted to take account of enumerated residents not expected to register with a local GP. Non-resident, term time students were added. Adjustment factors for estimated census undercoverage in different age-sex groups and geographical areas ${ }^{2}$ were applied to compare the number of registered patients with the best estimates of population from the census.

The estimated census coverage was $98 \cdot 1 \%$ in Norfolk and $98.4 \%$ in Suffolk. Comparing the total numbers of registered patients with adjusted census populations (table), the patient registers contained fewer children aged 0-4 years and (to a lesser extent) 5-14 years, but more people aged 15 and over. The highest

School of

Environmental

Sciences, University of

East Anglia, Norwich

NR4 7TJ England

R M Haynes

A A Lovett

G Bentham

J S Brainard

S H Gale

Correspondence to:

Dr R Haynes.

Accepted for publication

February 1995

f Epidemiol Community

Health 1995;49:440

Registered patients as a percentage of census populations adjusted for undercoverage estimates, by age and sex.

\begin{tabular}{lccccc}
\hline & \multicolumn{2}{l}{ Norfolk } & & \multicolumn{2}{c}{ Suffolk } \\
\cline { 2 - 3 } \cline { 5 - 6 } Age $(y)$ & Males & Females & & Males & Females \\
\hline $0-4$ & 92 & 92 & 91 & 91 \\
$5-14$ & 99 & 100 & 98 & 97 \\
$15-44$ & 103 & 102 & & 104 & 101 \\
$45-64$ & 101 & 100 & & 103 & 101 \\
$65-74$ & 101 & 101 & & 102 & 101 \\
$\geq 75$ & 103 & 101 & & 104 & 102 \\
All ages & 101 & 101 & 102 & 100 \\
\hline
\end{tabular}

consistent excesses in the registers were for men aged $15-44$ and 75 years and over.

Discussion: Smaller patient register totals than census estimates for the $0-4$ years age group can be explained by delays of several weeks in entering births in patient registers. The higher register totals for all ages over 15 years are consistent with general inflation of the registers in the range $0-4 \%$, caused by delays in deleting former patients no longer resident and deaths, with deaths probably a more significant factor in the 75 years and over group. The slightly larger difference between register and census estimates for males aged 15-44 might partly have been the result of including younger men no longer resident who did not register with a new doctor in another area, since OPCS figures showed a net outflow of persons aged 15-29 (but not other ages) from Norfolk and Suffolk FHSAs in 1991..$^{5}$ It is also possible that census undercoverage in excess of that allowed for may have been a contributory factor for both the 15-44 and over 74 years groups in males. Neither census undercoverage nor register inflation explains the slightly lower counts of children aged 5-14 years in the registers compared with the census.

These results suggest that patient register records might, after adjustment, provide population estimates that are broadly comparable with census estimates in census years and may be superior in the intercensal period for areas smaller than local authority districts. Estimates could be made for any small areas that can be built from unit postcodes. The patient register adjustment factors suggested by the averages from the table are 1.09 for ages $0-4$ years, 1.02 for ages 5-14 years, 0.99 for ages 45-74 years, and 0.98 for people aged $15-44$ and 75 years and over. We expect these findings to apply to other similar parts of the country but not to metropolitan areas, where non-registration and register inaccuracies are believed to pose greater problems. 